\title{
Understanding the role of $\mathrm{R} 266 \mathrm{~K}$ Mutation in Cystathionine $\beta$-synthase (CBS) Enzyme: An In Silico study
}

\author{
Aashish Bhatt and Md. Ehesan Ali* \\ Institute of Nano Science and Technology, Sector-81, Mohali, Punjab-140306 \\ India \\ *Email: $\underline{\text { ehesan.ali@inst.ac.in }}$
}

\begin{abstract}
Human cystathionine $\beta$-synthase (hCBS) is a Heme containing unique pyridoxal 5'-phosphate (PLP) dependent enzyme that catalyzes the bio-chemical condensation reactions in the transsulfuration pathway. The role of Heme in the catalytic activities of enzyme has not yet been understood completely, even though various experimental studies have indicated its participation in the bi-directional electronic communication with the PLP center. Most probably Heme acts as the electron density reservoir for the catalytic reaction center but not as a redox electron source. Here, in this work, we investigated In Silico dynamical aspects of the bi-directional communications by performing classical molecular dynamics (MD) simulations upon developing the necessary force field parameters for the cysteine and histidine bound hexacoordinated Heme. The comparative aspects of electron density overlap across the communicating pathways are also explored adopting the density functional theory (DFT) in conjunction with the hybrid exchange-correlation functional for the $\mathrm{CSB}^{\mathrm{WT}}$ (wild-type) and $\mathrm{CBS}^{\mathrm{R} 266 \mathrm{~K}}$ (mutated) case. The atomistic MD simulations and subsequent explorations of the electronic structures not only confirm the reported observations but provide an in-depth mechanistic understating of how the non-covalent hydrogen bonding interactions with Cys52 control such long-distance communication. Our study also provides a convincing answer to the reduced enzymatic activities in the R266K hCBS in comparison to the wild-type enzymes. We further realized that the difference in hydrogen-bonding patterns, as well as salt-bridge interactions, play a pivotal role in such long distant bi-directional communications.
\end{abstract}

Keywords: Heme Force Field, Allosteric Interactions, Heme PLP Communications, R266K Mutation, CBS Enzyme 


\section{Introduction}

Homocysteine is a sulfur-containing amino acid that plays an important role in cell homeostasis. ${ }^{1}$ High concentration of homocysteine in the body causes severe myopia, atherosclerosis (cardiovascular disease and stroke), intellectual disability etc. ${ }^{2,3}$ This condition develops due to the deficiency of the Cystathionine beta-synthase (CBS) enzymes, which convert homocysteine to cystathionine in the transsulfuration pathway of the methionine cycle. The CBS enzyme catalyses the bio-chemical condensation of homocysteine with serine (canonical activity) to form cystathionine in the transsulfuration pathway ${ }^{4}$ and produces $\mathrm{H}_{2} \mathrm{~S} .{ }^{5-}$

${ }^{8}$ In higher organisms, CBS is the only PLP-dependent enzyme that has a regulatory Heme cofactor. ${ }^{9,10}$ The general notion for human CBS (hCBS) enzyme is tetrameric in nature, however so far only dimeric crystal structures with missing domain or residues have been resolved. For example, the truncated hCBS lacks regulatory domain (PDB Id. 1JBQ) and hCBSdelta516-526 lacking 10 residues from the C-terminal domain (PDB Id. 4L0D, 4PCU etc). ${ }^{10-12}$ Each subunit of hCBS is divided into three parts; i.e. N-terminal heme-binding domain, catalytic domain or PLP-binding site, and a C-terminal regulatory domain or Sadenosyl methionine (SAM) binding domain. In N-terminal domain (NTD), Heme is solventexposed and hexacoordinated with Cys52 $\mathrm{S}^{-}$(thiolate) and $\mathrm{N} \varepsilon 2$ atom of His65 at the axial positions. ${ }^{10-12}$ The exact role of heme in hCBS enzyme is yet to be understood. In this context, Taoka et al. and Majtan et al. independently proposed the possible role of heme in the redox reaction as well as in protein folding. ${ }^{13,14}$ However, in absence of the NTD in the protein, the overall enzymatic activities is $\sim 20 \%$ of the complete wild-type hCBS. ${ }^{15}$ This indicates the participation of Heme in the enzymatic reaction that occurs in the PLP centre in the catalytic domain. PLP is covalently bound to protein via internal aldimine Lys119 and it is the catalytic centre for the substrate reaction. ${ }^{10-12,16}$ The reduction of the enzymatic activities of hCBS were reported when small molecules like NO, CO etc. binds to Heme in the ferrous state of iron. ${ }^{9,13}$ CO displaces the Cys52 from its binding state with heme and affects Arg266-Heme communication. On the other hand, NO makes Heme as penta-coordinated complex by attacking at His65 site and also eliminates Cys52 from it bindings site in Heme. In both the scenario it inhibits the bidirectional communications. ${ }^{9,17-19}$ The most striking fact is that the heme and PLP are located distant apart from each other by $20 \AA .{ }^{10-12}$

Based on the crystal structure of hCBS and several spectroscopic studies the bidirectional communication between the Heme and PLP sites was proposed to occur through the $\alpha$-helix 8 that interacts at one end with the Cys52(S-) Heme ligand via Arg266. ${ }^{10-12,17,20-24}$ 
(see Figure 1). Mutations in the conserved residues which binds the PLP catalytic center i.e. residue Thr-257 and Thr-260 disrupts the PLP-Heme communications and lowers the enzymatic activity compared to the wild-type enzyme. ${ }^{24}$ Smith et al. employing resonance Raman spectroscopy observed that the mutation in R266K invokes the geometric distortion into the heme due to Arg266-Cys52 interaction, that affects the PLP dependent activity as well. $^{22}$ (Figure 1) All these evidences indicate a complex inherent scenarios of interactions that control the CBS enzymatic activities. Therefore, it is inevitable to have an in-depth understanding for the communication between the Heme that acts as electron-reservoir being rich in quasi-degenerate $\pi$-electrons as well as d-electrons of the transition metal center, and the PLP in which enzymatic reaction takes place.

Molecular dynamics simulations for various Heme containing proteins are occasionally reported in the literature using different force field models. ${ }^{25-30}$ However to the best of our knowledge, the forcefields for hexa-coordinated Heme with cystine and histidine bound at the axials positions were never developed. In the hCBS, Doss and co-workers applying In Silico tools and MD simulations investigated the structure-functional insight into the effect of SNP on the stability and activity of mutant CBS. ${ }^{31}$ Later on Gupta et al. studied the G307S substitution in the catalytic function and protein conformation in the hCBS enzyme. ${ }^{32}$

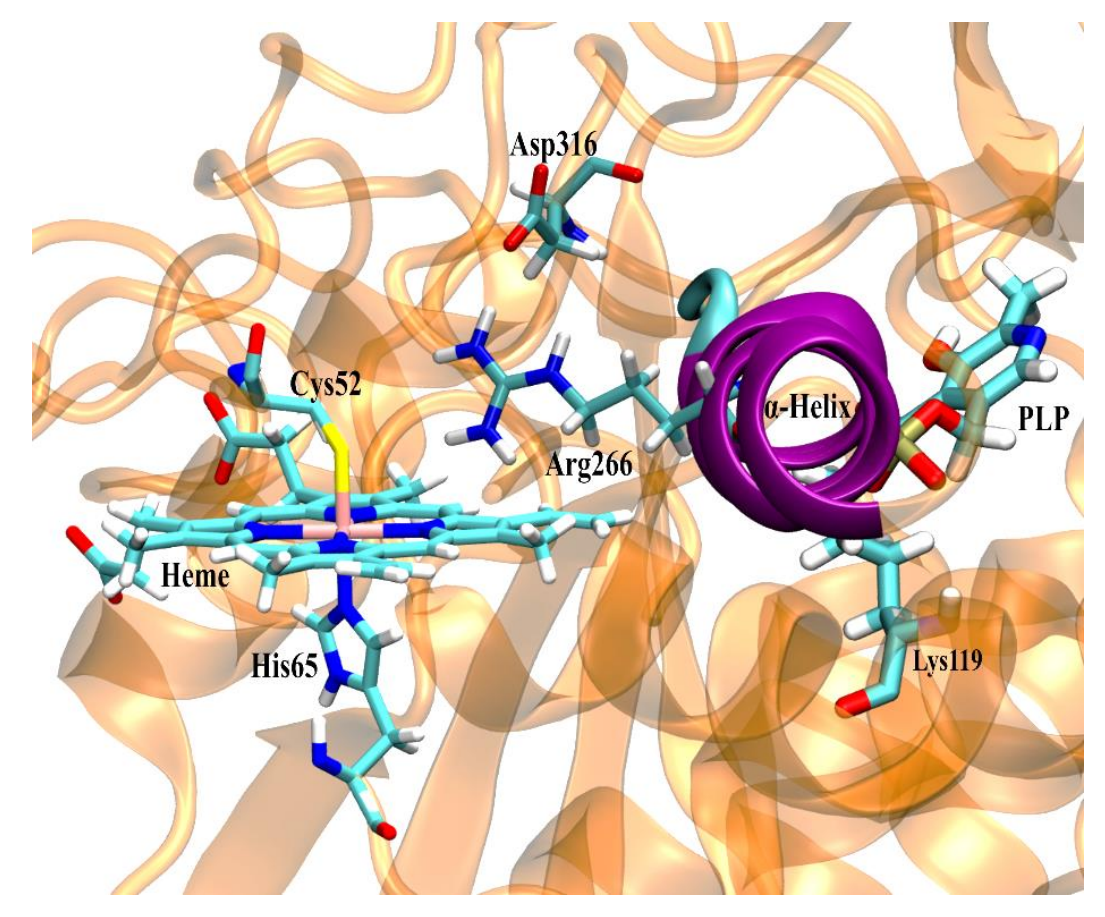

Figure 1. The cofactors and the key residues in hCBS enzyme that constitute the communication pathway between the Heme and PLP center. In the wild-type enzyme Arg266 forms strong non-covalent hydrogen-bond with the Cys52 as well as with the Asp316. Arg266 is covalently bound to $\alpha$-helix 8 that further binds with the PLP that acts as the catalytic center. 
In this study, we have investigated the effect of R266K mutation in hCBS enzyme employing the atomistic molecular dynamics (MD) simulation as well as density functional theory. More specifically, here we intended to understand the structural dynamics and the effect of mutation on the local change in the hydrogen bonding and salt-bridge interaction patterns and their impact on the electron communication channel between the Heme and the catalytic PLP centre. Further, we have explored the possible role of the Asp316 interactions in the Heme-PLP communications.

\section{Computational Methodology}

To perform the classical molecular dynamics simulations for human Cystathionine $\beta$-synthase (hCBS), the initial coordinates were obtained from the reported $\mathrm{x}$-ray protein crystal structure (PDB ID 4L0D). ${ }^{11}$ The R266K mutated structure was generated by mutating residue using Chimera 1.13.1. ${ }^{33}$ The $\mathrm{pK}_{\mathrm{a}}$ values of the individual amino acids were calculated on PDB2PQR server. ${ }^{34}$ A python-based metal centre parameter builder (MCPB.py Builder) was utilized to build the force fields for Heme, with Histidine and Cysteine residues as the axis ligands. ${ }^{35}$

The force fields for such Heme were developed upon a rigorous benchmarking process. The details of force field generation and its benchmarking studies are provided in the supporting information. In brief, the molecular geometry of the Heme along with the axial ligands were optimized using three different hybrids functional (viz. B3LYP, M06 and PBE0) within the framework of Density Functional Theory (DFT). The extraction of the force constants and the population analysis were performed using the optimized geometries. Antechamber in the AmberTools, were used to develop the PLP-Lys force field parameters (SI section 2).

To perform the MD simulation, the protein with cofactors has been explicitly solvated in a $10.0 \AA$ margin TIP3P water ${ }^{36}$ box and $12 \mathrm{Na}^{+}$ions are added to the system to make it charge neutralized. The complete simulation system except the hexacoordinated heme has been parameterized with the Amber ff14SB force field. ${ }^{37,38}$ Particle mess Ewald method ${ }^{39}$ has been used to account for the long rage interaction with a cut-off distance of $12 \AA$. NAMD 2.13 package was used to perform the classical MD simulations. ${ }^{40}$ Initially the system was equilibrated for $250 \mathrm{~ns}$ and subsequently $250 \mathrm{~ns}$ long trajectories were obtained as the production dynamics to perform the statistical analysis. Visualization and structural analysis were performed using VMD and Bio3 $\mathrm{d}^{41,42}$ The detailed descriptions for the computational methods adopted to perform the MD simulation are provided in SI (Sections 1 to 3). 


\section{Results and discussion}

To understand the role and impact of R266K mutation in biochemical activities of hCBS enzymes, we have systematically compared the simulated MD trajectories with the wild type (WT) hCBS and analysed them applying a number of statistical and computational tools. The details are discussed in the following sections.

\subsection{Comparison of structure and dynamics of the hCBS enzyme}

To obtain an intriguing understanding on the protein dynamics, the RMSDs of the structural fluctuations are compared between the $\mathrm{CBS}^{\mathrm{WT}}$ and $\mathrm{CBS}^{\mathrm{R} 266 \mathrm{~K}}$. A comparison of the backbone atoms $(\mathrm{C} \alpha, \mathrm{C}, \mathrm{N}$, and $\mathrm{O}) \mathrm{RMSD}$ of the CBS protein is illustrated in Figure $2 \mathrm{a}$ and $2 \mathrm{~b}$. The RMSD of the entire protein and its decomposition along the multiple domains are provided in Figure 2. The average RMSDs for the complete protein $\mathrm{CBS}^{\mathrm{WT}}$ and $\mathrm{CBS}^{\mathrm{R} 266 \mathrm{~K}}$ are found to be $3.38 \AA$ and $3.39 \AA$ respectively. The large fluctuation in the complete protein RMSD is primarily due to the intrinsically disordered regions of the $\mathrm{N}$-terminal end that fluctuate larger than the other part of the protein (see section in SI 3.2 and Figure S9). For catalytic domain, the average RMSD fluctuations for $\mathrm{CBS}^{\mathrm{WT}}$ is $\sim 1.30 \AA$ which is slightly larger than the mutated $\mathrm{CBS}^{\mathrm{R} 266 \mathrm{~K}}$ protein $(1.26 \AA)$. No significant difference is observed for the linker domains. However, it is observed that the C-terminal domain (CTD) has larger fluctuations in $\mathrm{CBS}^{\mathrm{WT}}$ as compared to the $\mathrm{CBS}^{\mathrm{R} 266 \mathrm{~K}}$. This indicates that mutation at the CTD domain provides confirmational stability for $\mathrm{CBS}^{\mathrm{R} 266 \mathrm{~K}}$ over the wild types.

The root-mean-square fluctuations (RMSF) of the catalytic domain and all the residues $\mathrm{C} \alpha$-atoms (CA) illustrated in Figure 2c and in SI Figure S10 reveals that in the catalytic domain several residues associated with R266K mutation (represented by a vertical dotted line) show significant differences in the RMS fluctuations. In this particular mutation, the protein structure exhibits a systematic lower RMSF fluctuation compared to the wild-type enzyme. This can be interpreted as a more compact protein structure for the mutated case. This might be a consequence of the smaller spatial volume occupied by the linear Lys266 as compared to the branched Arg266 residue. In addition to that, Lys266-Asp316 has strong salt-bridge interaction compared to Arg266-Asp316. (See in Figure S11) Thus, Asp316 restricts the atomistic motions of the Lys266 compared to Arg266. This is why CBS ${ }^{\mathrm{WT}}$ shows higher RMSF compare to $\mathrm{CBS}^{\mathrm{R} 266 \mathrm{~K}}$. This fact indicates that a single mutation in the protein has a remarkable impact on the overall structure and dynamics of the protein. The mutation even influences the atomistic motions of the residues those are quite far from the mutation sites. 

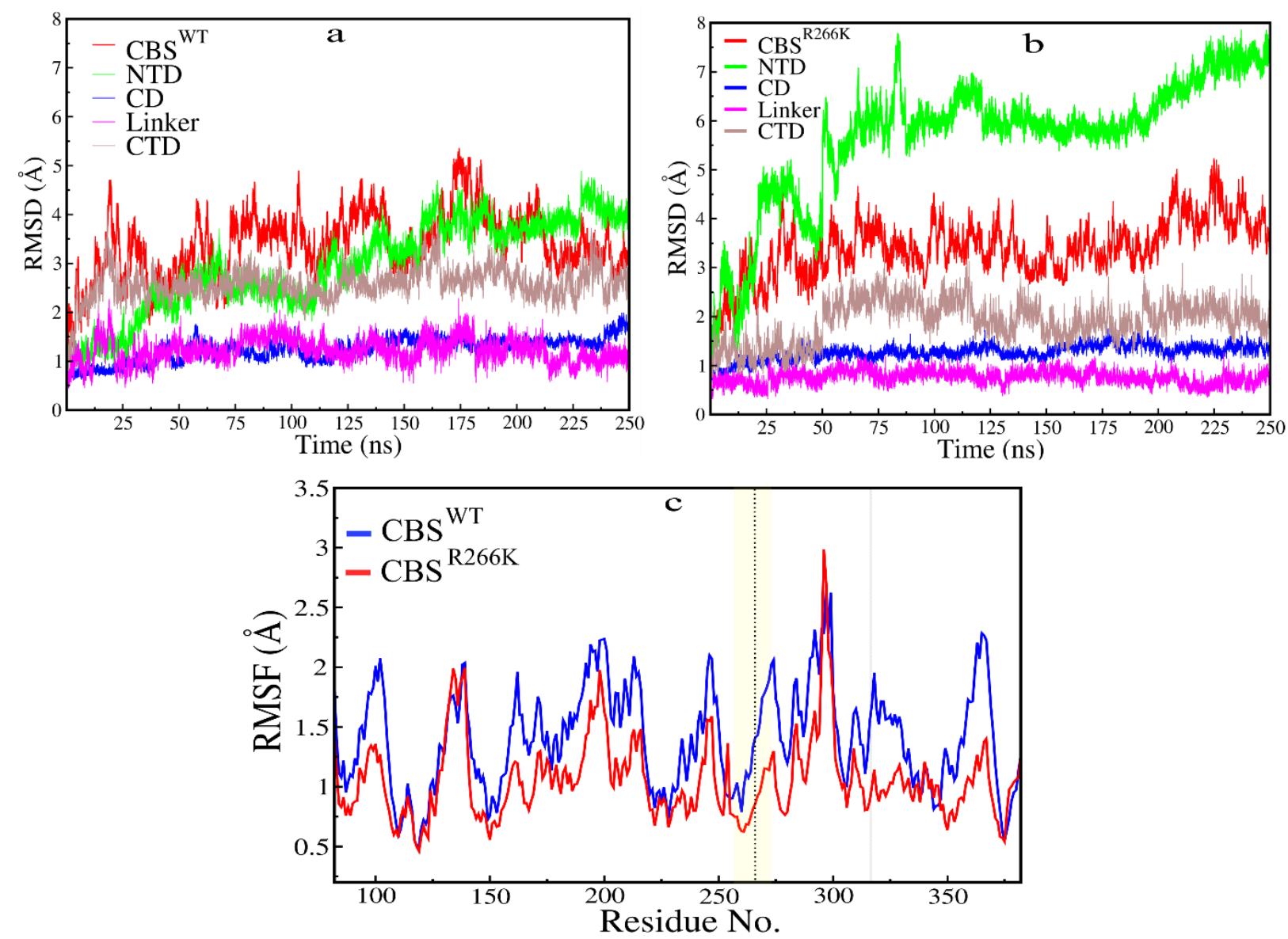

Figure 2. Calculated RMSD of (a) $\mathrm{CBS}^{\mathrm{WT}}$ and (b) $\mathrm{CBS}^{\mathrm{R} 266 \mathrm{~K}}$ with a different domain. Where red colour shows the RMSD of complete protein (1-542 aa), green is for N-terminal Domain (NTD) (1 to 70 aa), blue is for Catalytic Domain (CD) (71 to 381 aa), magenta is for linker (382 to 412 aa) and Brown is for C-Terminal Domain (CTD) or regulatory domain (412 to 542 aa). (c) Catalytic domain comparisons of the RMSF for $\mathrm{CBS}^{\mathrm{WT}}$ and $\mathrm{CBS}^{\mathrm{R} 266 \mathrm{~K}}$.

\subsection{Dynamic Cross-correlation Matrix (DCCM) \& Allosteric Interactions}

In the previous section, we realized that the impact of the structural fluctuations due to a single mutation in the protein is not localized or restricted within the mutated site itself, rather its impact has a long-range consequence on the residues even far apart from the mutated sites. For a deeper understanding of how does such impact gets transmitted in the long distances within the protein, we have performed the dynamical cross-correlation analysis by computing all the pairwise Pearson cross-correlation coefficients for the $\mathrm{C} \alpha$-atoms of all the residues. The crosscorrelation matrix (Figure 3) provides a coarse and prompt picture of the most relevant linearly coupled motions occurring across the complete protein during the MD simulations. 

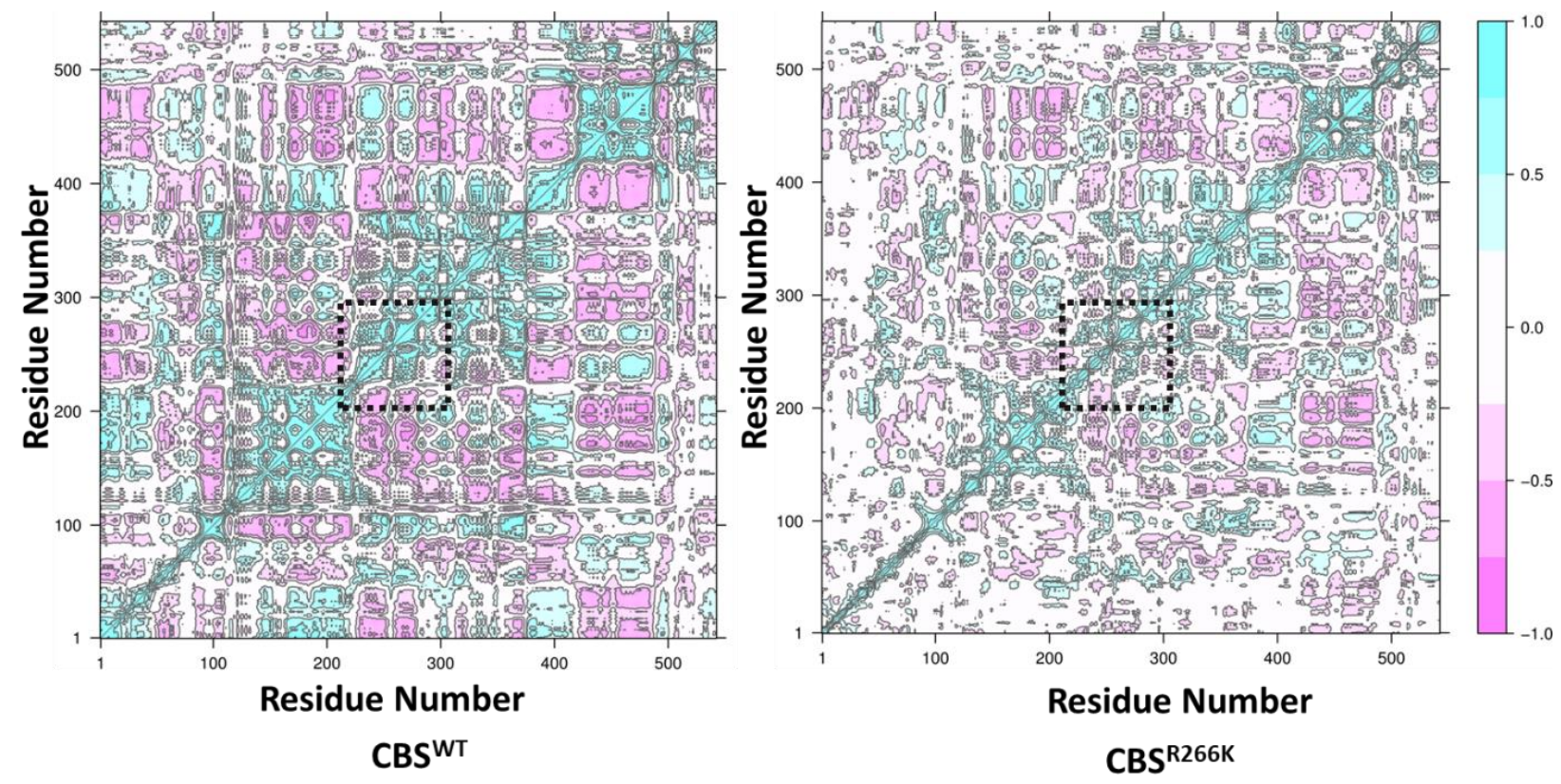

Figure 3. The Dynamic cross-correlation (DCCM) maps for the hCBS proteins obtained from the entire production run trajectories. The region of the black dotted circle indicates the region of the mutated site that has a strong positive correlation in the $\mathrm{CBS}^{\mathrm{WT}}$. Colour Code: cyan colour bar belongs to the (positive) correlation, magenta is for anti-correlation and white depicts no correlation at all.

The dynamical cross-correlations analysis reveals that the atomistic motions are strongly correlated in $\mathrm{CBS}^{\mathrm{WT}}$ compared to the $\mathrm{CBS}^{\mathrm{R} 266 \mathrm{~K}}$, even though the RMSD and RMSF fluctuations for the former one is quite larger as compared to mutated case. It is quite evident from the DCCM map in Figure 3, that for $\mathrm{CBS}^{\mathrm{WT}}$ protein residues are far apart from each other and having quite strong correlations (either positive or negative), but for $\mathrm{CBS}^{\mathrm{R} 266 \mathrm{~K}}$ the uncorrelated motions (as depicted by white space in Figure 3) predominate over the correlated motions of the residues. We trace down this as the change in the non-bonded interactions (see SI section 3.4 and Figure S12). A strong hydrogen bonded network between Cys52, Arg266, and Asp316 is present in $\mathrm{CBS}^{\mathrm{WT}}$ protein, however, mutation weakens these hydrogen-bonding patterns in the mutated $\mathrm{CBS}^{\mathrm{R} 266 \mathrm{~K}}$ protein. We also find strong salt bridge interactions between the Asp316 and Lys266 compared with Arg266. All these non-covalent interactions contribute to the strong correlations pattern for the wild-type enzyme compared to the mutated case. Hence it is quite evident that the mutation R266K drastically reduces the correlations in atomistic motions especially between long-distant residues. Such a huge change in the atomistic motions occurs just for a single mutation. This clearly indicates that the mutations breaks the long-distance communications and reduces the allosteric response in the protein dynamics. Hence the reduced enzymatic activities in the mutated protein could be correlated 
as the lack of communications due to the uncorrelated atomistic motions compared to the Wildtype enzyme.

\subsection{Long-range Communications between Heme and Catalytic Domain}

In the context of Long-range communications, Singh et al. established that the Heme, residing $\sim 20 \AA$ apart from the PLP, allosterically controls the enzymatic activities in the catalytic center 17. Here to obtain atomistic and electronic details of such long-range communications the MD simulation trajectories are investigated in detail. It reveals quite a remarkable difference in the atomistic motions and electronic structures especially due to the R266K mutations. As illustrated in Fig. 2a, Arg266 interacts with the Heme through a strong hydrogen bond with Cys52. The probability distribution of the distance between the Cys52-Arg266 is shown in Figures $4 \mathrm{a}$ and c. The maxima of the distributions appeared at $2.28 \AA$ for the wild type hCBS(Magenta curve in Figure 4c). The singular peak for the wild-type hCBS confirms stable and strong hydrogen-bond formation with the rigid structural orientation of the Arg266 due to hydrogen bonds with Cys52 as well as Asp316. However, the hydrogen-bond formation pattern changes remarkably due to the R266K mutations (shown with green colour in Figure 4b and c).

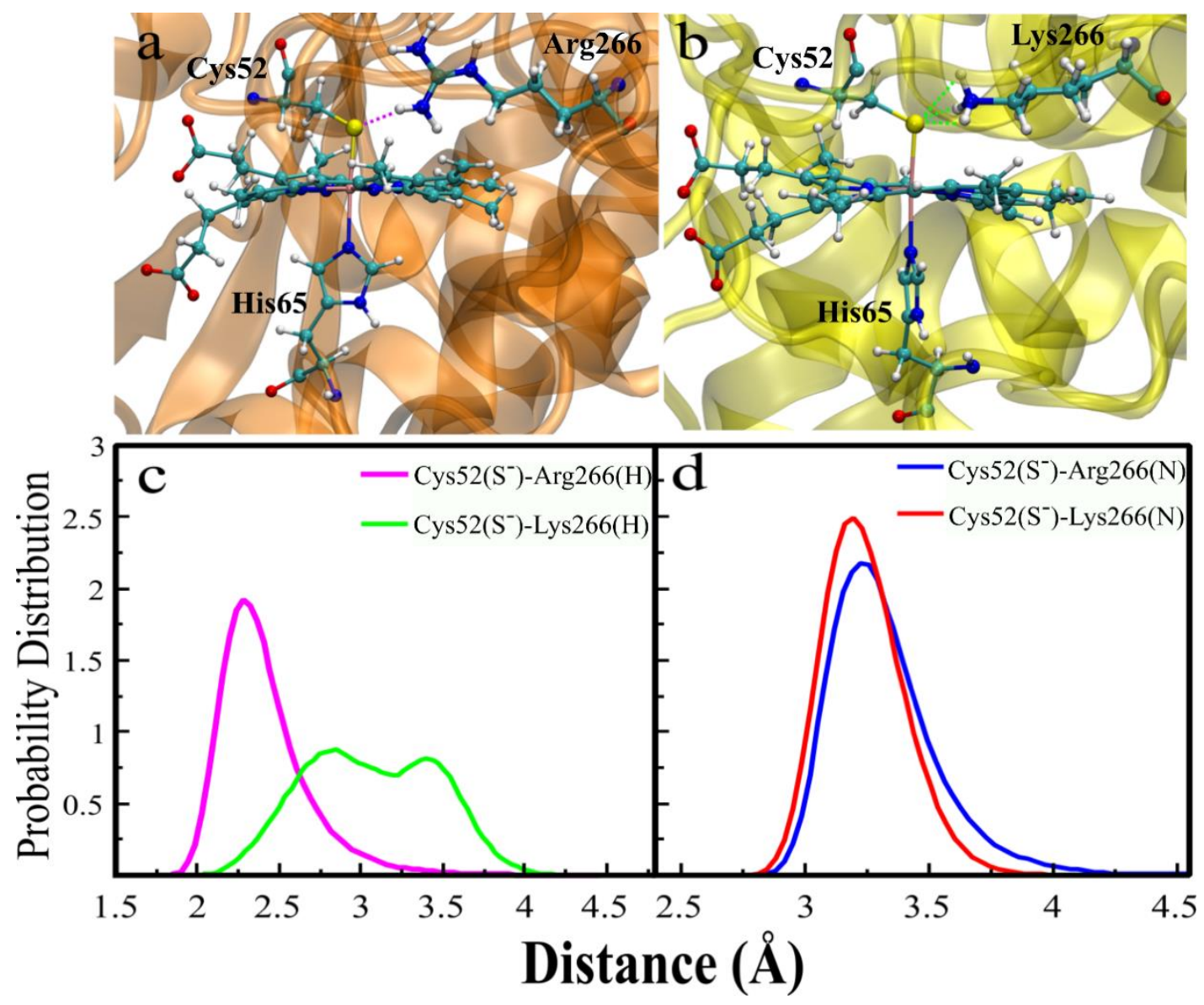




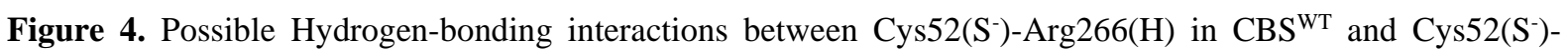
Lys266 in $\mathrm{CBS}^{\mathrm{R} 266 \mathrm{~K}}$ are depicted in the upper panel (a) and (b) respectively. The probability distributions of these hydrogen bonds Cys52(S-)-Arg266/Lys266(H) along with the distance between the Cys52(S-)-Arg266/Lys266(N) are shown in the bottom panel (c) and (d) respectively. The dual peaks for the mutated case (green curve) in (c) indicate that the hydrogen atoms responsible for these interactions (shown in Figure 4b) with the Heme are in continuous rotational motions.

The probability distribution of Cys52( $\left.\mathrm{S}^{-}\right)$-Lys266(H) distance along the MD trajectory (Figure 4c) results in a broader distribution with the appearance of two peaks at $2.81 \AA$ and $3.38 \AA$. This indicates the hydrogen-bond formation in the mutated case is quite weak (or more precisely "less probable") compared to the wild-type hCBS. However, a decreasing trend of RMSF (Figure 2c) in the case of R266K indicates the weakening of the Hydrogen bonding interactions resulted in diminished movements of the $\mathrm{C} \alpha$ atoms. This fact is further supported by the loss of strongly correlated motion of the selected residues (see Figure SI12) in R266K. As a consequence of these alterations in the dynamic properties of the R266K mutations, the transmission of allosteric communications between the Heme and the catalytic PLP center has decreased.

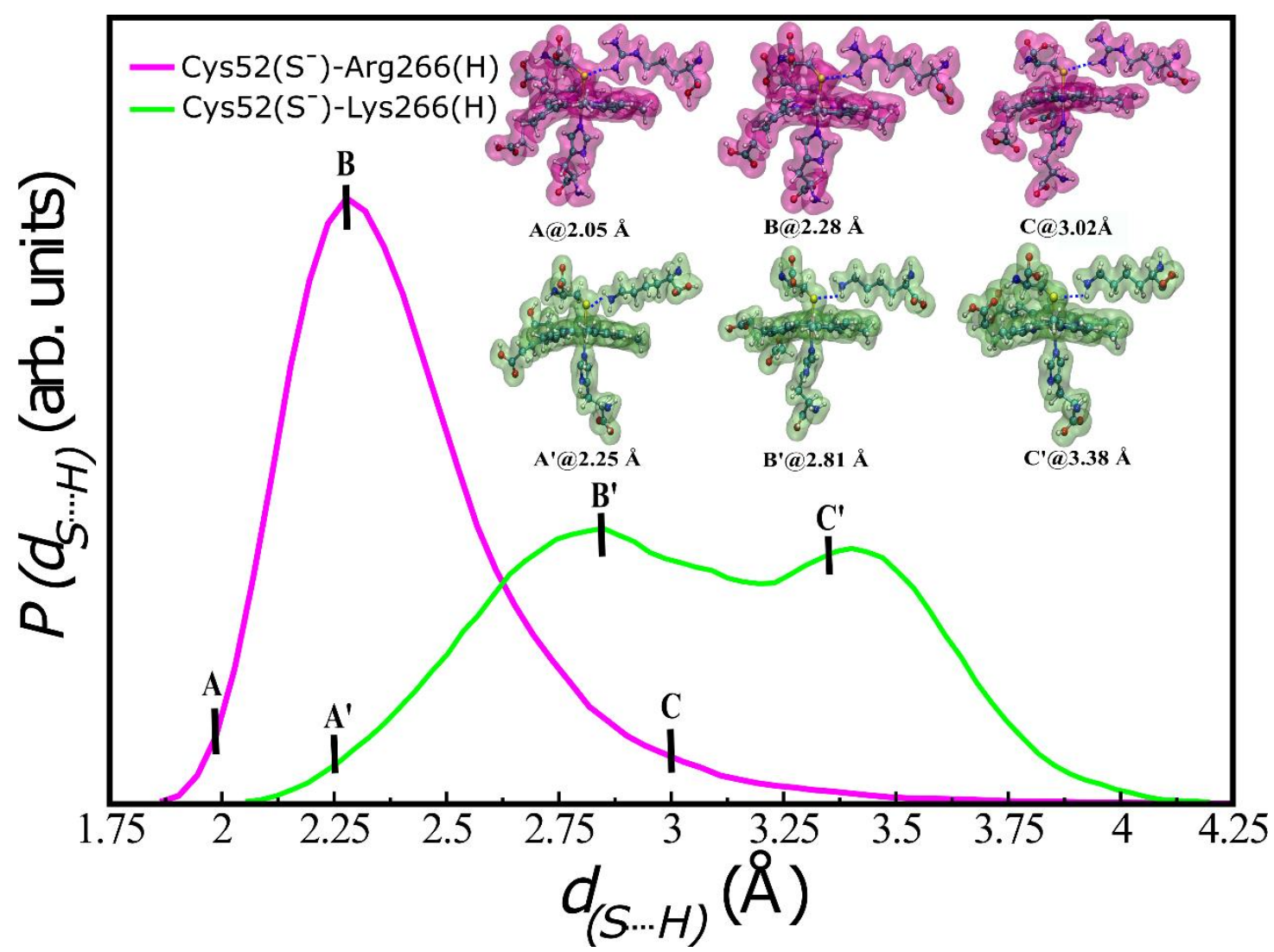

Figure 5. The electron density obtained as computed employing DFT (PBE0/6-31G*) level calculations (isosurface value $0.025 \mathrm{e}^{-/} \AA^{3}$ ) at the configurations with the selected distances as indicated in the probability distribution curve of hydrogen-bonding between Cys52-Arg/Lys266. The selected H-bonding distances for 
Arg266 with Cys52 are indicated as A@2.05 A, B@2.28 (most probable value), C@3.01 Lys266 are indicated as A'@2.25 ̊, B'@2.81 $($ most probable value), C'@3.38

Figure $4 \mathrm{~d}$ reveals an interesting fact about the distance between Cys52( $\left.\mathrm{S}^{-}\right)$and

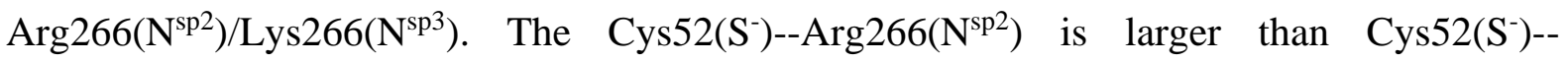
Lys266( $\left.\mathrm{N}^{\mathrm{sp} 3}\right)$, but Arg266 forms strong hydrogen bonding due to $\mathrm{sp}^{2}$ hybridization with Cys52 $\left(\mathrm{S}^{-}\right)$amino acid residue in the hCBS enzyme.

To get a deeper understanding of the non-bonding interactions we have further investigated the electron density distribution computed with PBE0/6-31G* (shown in Figure 5) and CAM-B3LYP/6-31G* (shown in SI, Figure S13) within the long-range correction hybrid density functional theory (DFT) framework. We have adopted a few distinct snapshots from all over the distribution (Figure 5). It is quite evident that for the wild type hCBS, at the most probable distance $(\sim 2.28 \AA)$, there is a strong overlap in the electron density between the interacting residues; Cys52 and Arg266. However, no such overlap in the electron density is observed at the most probable configuration for the R266K mutated case (Figure 5). Thus, it is quite evident that the R266K mutations not only break the strong hydrogen-bonding pattern but also modulate electronic communications.

\subsection{Role of Asp316, $\alpha$-helix 8 and PLP Binding sites in the bidirectional Communications}

It is now quite evident that the Cys52( $\left.\mathrm{S}^{-}\right)-\operatorname{Arg} 266(\mathrm{H})$ hydrogen-bond interaction is the key contributor to the long-distance bi-directional electronic communications between the Heme and PLP catalytic center. Thus, disruption of this interaction affects the hCBS enzymatic activities. Furthermore, the analysis of the MD trajectories revealed that Asp316 residue plays a pivotal role in such long-distant interactions as well by providing a favorable environment for the Arg266, which manifests a stable and strong non-covalent hydrogen-bonding with the Cys52( $\left(\mathrm{S}^{-}\right)$. The de-protonated anionic oxygen in the side chain of Asp316 formed a strong hydrogen bonding with the neighboring residue especially with the Arg266 in CBS ${ }^{\mathrm{WT}}$ (shown in Figure 6a) and Lys266 in $\mathrm{CBS}^{\mathrm{R} 266 \mathrm{~K}}$ (shown in Figure 6d).

The probability distributions of the hydrogen bonding between Asp316 and Arg266 in CBS WT as well as Asp316 and Lys266 in $\mathrm{CBS}^{\mathrm{R} 266 \mathrm{~K}}$ revealed two peaks for the wild-type and three for the mutated cases (Figure 6b). This indicates that two types of hydrogen bonding are possible for the wild-type, while three for the mutated cases appear due to rotational switching of the $\mathrm{H}$-atoms present in Lys266 in the $\mathrm{CBS}^{\mathrm{R} 266 \mathrm{~K}}$. Further investigation of salt-bridge analysis 
(Figure 6c) clearly indicate a rigid and compact structure of Lys266 in $\mathrm{CBS}^{\mathrm{R} 266 \mathrm{~K}}$ as one can also see from RMSF (Figure 2c).
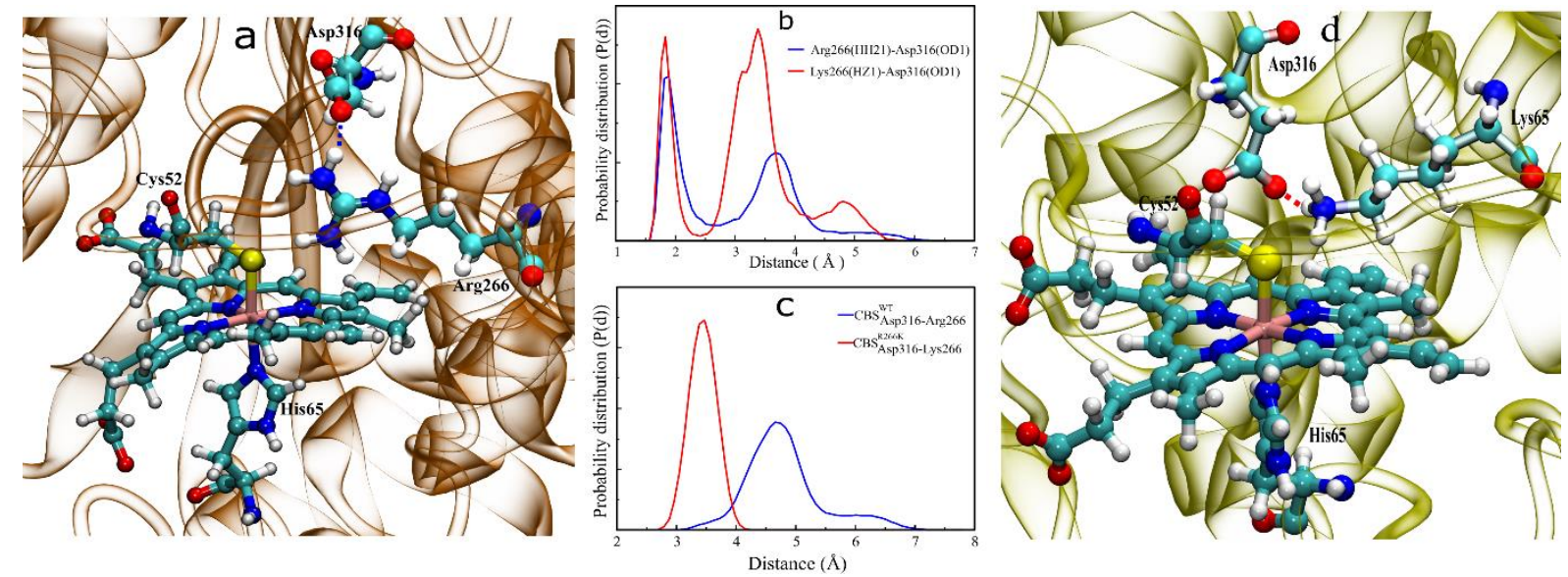

Figure 6. Non-covalent interaction between Asp316 and Arg266/Lys266 in the CBS ${ }^{\mathrm{WT}}$ and CBS ${ }^{\mathrm{R} 266 \mathrm{~K}}$ system. (a) Graphical representation of $\mathrm{CBS}^{\mathrm{WT}}$ with selective residue. b) Probability distribution of the hydrogen bonds between Asp316(O)A-rg266(H)(Blue colour) and Asp316(O)-Lys266(H)(Red colour). c) Salt-bridge interactions between Asp316-Arg266(Blue) and Asp316-Lys266(Red). d) Graphical representation of Asp316 interaction with the Lys266 in the $\mathrm{CBS}^{\mathrm{R} 266 \mathrm{~K}}$ system.

This could be inferred from the sharp peak of salt-bridge interactions in Figure 6c compared to the Wild-type enzyme (see section 3.3 in SI and figure S11). Despite the mutated CBS ${ }^{\mathrm{R} 266 \mathrm{~K}}$ enzyme being compact and rigid, the intramolecular hydrogen-bonding interactions are quite weak in the mutated case as compared to the wild-type for the Cys52-Lys266. Although, there is no direct evidence of direct participation of the Asp316 in bidirectional communication, but it is evident that Asp316 stabilizes Arg266 in CBS ${ }^{\mathrm{WT}}$. Asp316 is a part of the catalytic loop which enclosed the catalytic centre and indirectly influenced the catalytic cavity. Thus, Asp316 might play an important role in conformational flexibility necessary for the substrate translocation to the catalytic centre. On the other hand, $\alpha$-helix 8 is a common communicator between Heme and the PLP site which contain Arg266/Lys266 of the protein. Thus, it is a part of the communication pathway between heme and the PLP site. The comparison of the RMSD between $\mathrm{CBS}^{\mathrm{WT}}$ and $\mathrm{CBS}^{\mathrm{R} 266 \mathrm{~K}}$ (SI section 3.6 and Figure $\mathrm{S} 14$ ) reveals that $\alpha$-helix 8 in $\mathrm{CBS}^{\mathrm{WT}}$ protein is slightly more compact compared to the mutated case.

It has been reported that R266K exhibits different enzymatic activity for different substrates 22,23 . The question is still there if there is any significant local structural and dynamical impact on the catalytic PLP-binding domain that might be impacting the enzymatic activities? Comparing the PLP binding sites between the Wild type and mutated case it is quite evident that there is no significant change is observed between the PLP and corresponding bindings 
sites (residue Thr257 \& Thr260) of the enzyme (see SI section 3.7). It is, thus, evident that the R266K mutation does not affect the PLP phosphate-binding site.

\subsection{Conformational rigidity in the catalytic center}

The catalytic center or PLP exposed sites are enclosed through the Loops L145-148, L171-174, L191-202, and L295-316 aa residues in the hCBS enzyme. Upon careful investigation of the trajectory snapshots (Figure 7), it is observed that $\mathrm{CBS}^{\mathrm{WT}}$ has larger exposure to the catalytic

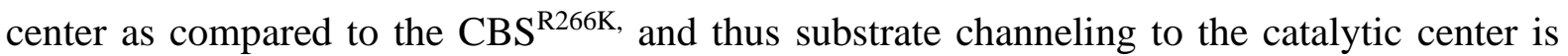
more feasible in the wild-type compared to the mutated one. This could also be attributed to the increased rigidity and compactness of the structure due to Lys266. This lost flexibility of the enzyme hinders the substrate channeling to the catalytic center, and thus loses the enzymatic activity in the mutated enzyme. ${ }^{43}$

To have an insight into the CTD motion, we analyzed the deviation of the MD trajectory by superimposing the final snapshots for $\mathrm{CBS}^{\mathrm{WT}}$ and $\mathrm{CBS}^{\mathrm{R} 266 \mathrm{~K}}$ with the crystal structure, which depicted the CTD orientation in both $\mathrm{CBS}^{\mathrm{WT}}$ and $\mathrm{CBS}^{\mathrm{R} 266 \mathrm{~K}}$ system (See section SI 3.8 and figure S17).

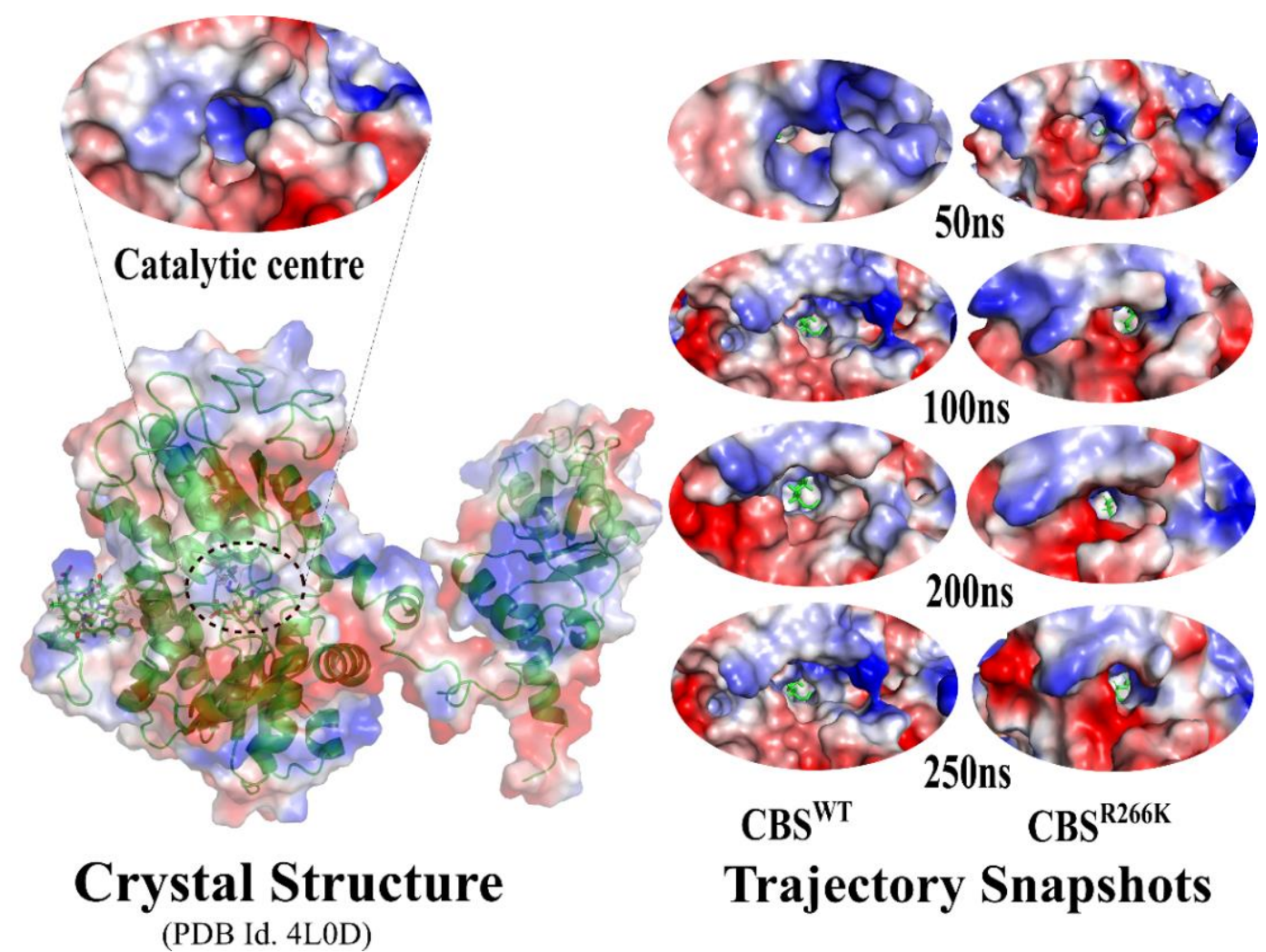

Figure 7. Electrostatic potential surface of the Catalytic centre in the catalytic domain at the hCBS enzyme.

Trajectory snapshots of the selected snapshots of the catalytic centre. 


\section{Conclusions}

We have compared the structural dynamics of the hCBS enzyme employing the classical molecular dynamics (MD) simulations for wild-type (hCBS ${ }^{\mathrm{WT}}$ ) and the mutated (hCBS ${ }^{\mathrm{R} 266 \mathrm{~K}}$ ) one. The hCBS contains a Heme cofactor, which is attached to the protein through axial coordination bonding with one cysteine and one histidine. The hexacoordinated Heme is in the low spin-state (singlet), for which the forcefield parameters are explicitly developed and benchmarked with the various density functional theory-based static calculations. Our simulations captured the long-range allosteric interactions between the Heme and the catalytic PLP center and confirm the previous experimental observations. In this work, we have realized that the Arg266 interacts strongly with the Heme co-factor through a strong hydrogen bond with Cys52 residue. The singular peak in the probability distribution curve of the particular hydrogen bonding (Cys52-Asrg266) confirms the stable and strong hydrogen-bond formation with a rigid structural motif of the Arg266 due to hydrogen bonds with Cys52 as well as with Asp316. The R266K mutation invokes disruptions to this stable structural motif and also reduces the overlap of the electronic states. Hence, this is the primary reason behind the decrease of allosteric communications between the Heme and the catalytic PLP center that has also been captured in the DCCM patterns due to the R266K mutations that reduces the enzymatic activities. The R266K mutations not only break the strong hydrogen-bonding pattern but also modulate electronic communications. In the long-distance interactions between the heme and catalytic center, the non-covalent hydrogen bonding between Cys52( $\left.\mathrm{S}^{-}\right)-\operatorname{Arg} 266(\mathrm{H})$ plays a key role, and breaking of this interaction causes the loss of enzymatic activities.

\section{Acknowledgments}

The authors thank Aritra Mukhopadhyaya, Ashima Bajaj and Rehan Khan for various helpful discussions. We acknowledge the financial support from the Department of Science and Technology through SERB-ECR project no. ECR/2016/000362 and Indo-Sweden joint project no. DST/INT/SWD/VR/P-01/2016. The Ph.D. fellowship support and computational resources provided by the Institute of Nano Science and Technology (INST) is thankfully acknowledged.

\section{Supporting Information}

Details of Heme force field parameter generation and benchmarking, PLP force field preparation, structural parameters comparison for $\mathrm{CBS}^{\mathrm{WT}}$ and $\mathrm{CBS}^{\mathrm{R} 266 \mathrm{~K}}$.

\section{Disclosure statement}

The authors declare no competing interests 


\section{References}

1. Fowler, B., Disorders of homocysteine metabolism. J. Inherit. Metab. Dis. 1997, 20, 270-85.

2. de Koning, A.B.; Werstuck, G.H.; Zhou, J.; Austin, R.C., Hyperhomocysteinemia and its role in the development of atherosclerosis. Clin. Biochem. 2003, 36, 431-41.

3. Majtan, T.; Pey, A.L.; Ereño-Orbea, J.; Martínez-Cruz, L.A.; Kraus, J.P., Targeting Cystathionine Beta-Synthase Misfolding in Homocystinuria by Small Ligands: State of the Art and Future Directions. Curr. Drug. Targets. 2016, 17, 1455-70.

4. Singh, S.; Padovani, D.; Leslie, R.A.; Chiku, T.; Banerjee, R., Relative Contributions of Cystathionine $\beta$-Synthase and $\gamma$-Cystathionase to $\mathrm{H}_{2} \mathrm{~S}$ Biogenesis via Alternative Trans-sulfuration Reactions. J. Biol. Chem. 2009, 284, 22457-66.

5. Braunstein, A.E.; Goryachenkova, E.V.; Tolosa, E.A.; Willhardt, I.H.; Yefremova, L.L., Specificity and some other properties of liver serine sulphhydrase: Evidence for its identity with cystathionine $\beta$-synthase. Biochimica et Biophysica Acta. 1971, 242, 247-260.

6. Papadopoulos, A.I.; Walker, J.; Barrett, J., A novel cystathionine Beta-synthase from Panagrellus redivivus (Nematoda). Int J Biochem Cell Biol. 1996, 28, 543-549.

7. Walker, J.; Barrett, J.; Thong, K.W., The identification of a variant form of cystathionine Beta-synthase in nematodes. Exp. Parasitol. 1992, 75, 415-424.

8. Majtan, T.; Krijt, J.; Sokolová, J.; Křǐžková.; M.; Ralat. M.A.; Kent. J.; Gregory, J.F.; Kožich, V.; Kraus. J.P., Biogenesis of Hydrogen Sulfide and Thioethers by Cystathionine Beta-Synthase. Antioxid. Redox. Signal. 2018, 28, 311-323.

9. Taoka, S.; West, M.; Banerjee, R., Characterization of the Heme and Pyridoxal Phosphate Cofactors of Human Cystathionine $\beta$-Synthase Reveals Nonequivalent Active Sites. Biochemistry. 2009, 38, 2738-2744.

10. Meier, M.; Janosik, M.; Kery, V.; Kraus, J.P.; Burkhard, P. Structure of human cystathionine beta-synthase: a unique pyridoxal 5'-phosphate-dependent heme protein. EMBO. J. 2001, 20, 3910-6.

11. Ereño-Orbea, J.; Majtan, T.; Oyenarte, I.; Kraus, J.P.; Martínez-Cruz, L.A., Structural basis of regulation and oligomerization of human cystathionine $\beta$-synthase, the central enzyme of transsulfuration. Proc. Natl. Acad. Sci. U. S. A. 2013, 110, E3790-9.

12. Ereño-Orbea, J.; Majtan, T.; Oyenarte, I.; Kraus, J.P.; Martínez-Cruz, L.A.Structural insight into the molecular mechanism of allosteric activation of human cystathionine $\beta$ - 
synthase by S-adenosylmethionine. Proc. Natl. Acad. Sci. U. S. A. 2014, 111, E384552.

13. Taoka, S.; Ohja, S.; Shan, X.; Kruger, W.D.; Banerjee, R., Evidence for heme mediated redox regulation of human cystathionine beta-synthase activity. J. Biol. Chem. 1998, $273,25179-84$.

14. Majtan, T.; Singh, L.R.; Wang, L.; Kruger, W.D.; Kraus, J.P., Active Cystathionine $\beta$ Synthase Can Be Expressed in Heme-free Systems in the Presence of Metal-substituted Porphyrins or a Chemical Chaperone. J. Biol. Chem. 2008, 283, 34588-34595.

15. Oliveriusová, J.; Kery, V.; Maclean, K.N.; Kraus, J.P., Deletion mutagenesis of human cystathionine beta-synthase. Impact on activity, oligomeric status, and Sadenosylmethionine regulation. J. Biol. Chem. 2002, 277, 48386-94.

16. Kery, V.; Poneleit, L.; Meyer, J.D.; Manning, M.C.; Kraus, J.P., Binding of pyridoxal 5 '-phosphate to the heme protein human cystathionine beta-synthase. Biochemistry. 1999, 38, 2716-24.

17. Singh, S.; Madzelan, P.; Stasser, J.; Weeks, C.L.; Becker, D.; Spiro, T.G.; Penner-Hahn, J.; Banerjee, R., Modulation of the heme electronic structure and cystathionine $\beta$ synthase activity by second coordination sphere ligands: The role of heme ligand switching in redox regulation. J. Inorg. Biochem. 2009, 103, 689-697.

18. Gherasim, C.; Yadav, P.K.; Kabil, O.; Niu, W.; Banerjee, R., Nitrite Reductase Activity and Inhibition of $\mathrm{H}_{2} \mathrm{~S}$ Biogenesis by Human Cystathionine B-Synthase. Plos. One. 2014, 9, e85544.

19. Vicente, J.B.; Colac, H.G.; Mendes, M.I.S.; Sarti, P.; Leandro, P.; Giuffre, A., NO* Binds Human Cystathionine B-Synthase Quickly and Tightly. J. Biol. Chem. 2014, 289, 8579-87.

20. Singh, S.; Madzelan, P.; Banerjee, R., Properties of an unusual heme cofactor in PLPdependent cystathionine $\beta$-synthase. Nat. Prod. Rep. 2007, 24, 631-639.

21. Weeks, C.L.; Singh, S.; Madzelan, P.; Banerjee, R.; Spiro, T.G., Heme regulation of human cystathionine $\beta$-synthase activity: Insights from fluorescence and Raman spectroscopy. J. Am. Chem. Soc. 2009, 131, 12809-12816.

22. Smith, A.T.; Su, Y.; Stevens, D.J.; Majtan, T.; Kraus, J.P.; Burstyn, J.N., Effect of the Disease-Causing R266K Mutation on the Heme and PLP Environments of Human Cystathionine $\beta$-Synthase. Biochemistry. 2012, 51, 6360-6370. 
23. Majtan, T.; Kraus, J.P., Folding and activity of mutant cystathionine $\beta$-synthase depends on the position and nature of the purification tag: characterization of the R266K CBS mutant. Protein Expr Purif. 2012, 82, 317-24.

24. Yadav, P.K.; Xie, P.; Banerjee, R., Allosteric communication between the PLP and heme sites in the $\mathrm{H}_{2} \mathrm{~S}$-generator human cystathionine $\beta$-synthase. J. Biol. Chem. 2012, 287, 37611-37620.

25. Cornell, W.D.; Cieplak, P.; Bayly, C.I.; Gould, I,R.; Merz, K.M.; Ferguson, D.M.; Spellmeyer, D.C.; Fox, T.; Caldwell, J.W.; Kollman, P.A., A Second-Generation Force Field for the Simulation of Proteins, Nucleic Acids, and Organic Molecules. J. Am. Chem. Soc. 1995, 117, 5179-5197.

26. MacKerell Jr, A. D.; Bashford, D.; Bellott, M.; Dunbrack Jr, R. L.; Evanseck, J. D.;Field, M. J.; Fischer, S.; Gao, J.; Guo, H.; Ha, S. et al. All-atom empirical potential for molecular modeling and dynamics studies of proteins. J. Phys. Chem. B. 1998, 102, $3586-3616$.

27. Autenrieth, F.; Tajkhorshid, E.; Baudry, J.; Luthey-Schulten, Z., Classical force field parameters for the heme prosthetic group of cytochrome c. J. Comput. Chem. 2004, 25, 1613-22.

28. Oda, A.; Yamaotsu, N.; Hirono, S., New AMBER Force Field Parameters of Heme Iron for Cytochrome P450s Determined by Quantum Chemical Calculations of Simplified Models. J. Comput. Chem. 2005, 26, 818-826.

29. Chillemi, G.; Anselmi., M.; Sanna, N.; Padrin, C.; Balducci, L.; Cammarata, M.; Pace, E.; Chergui, M.; Benfatto., M., Dynamic multiple-scattering treatment of X-ray absorption: Parameterization of a new molecular dynamics force field for myoglobin. Struct. Dyn. 2018, 5, 054101.

30. Mustafa, G.; Nandekar, P.P.;Mukherjee, G.; Bruce, N.J.; Wade, R.C., The Effect of Force-Field Parameters on Cytochrome P450-Membrane Interactions: Structure and Dynamics. Sci. Rep. 2020, 10, 7284.

31. Doss, C.G.P.; Rajith, B.; Magesh, R.; Kumar, A.A., Influence of the SNPs on the structural stability of CBS protein: Insight from molecular dynamics simulations. Front. Bio. 2014, 9, 505-518.

32. Gupta, S.; Kelow, S.; Wang, L.; Andrake, M.D.; Roland L. Dunbrack Jr, R.L.; Kruger, W.D.,Mouse modeling and structural analysis of the p.G307S mutation in human 
cystathionine $\beta$-synthase $(C B S)$ reveal effects on CBS activity but not stability. J. Biol. Chem. 2018, 293, 13921-13931.

33. Pettersen, E.F.; Goddard, T.D.; Huang, C.C.; Couch, G.S.; Greenblatt, D.M.; Meng, E.C.; Ferrin, T.E., UCSF Chimera--a visualization system for exploratory research and analysis. J. Comput. Chem. 2004, 25, 1605-12.

34. Dolinsky, T.J.; Czodrowski, P.; Li, H.; Nielsen, J.E.; Jensen, J.H.; Klebe, G.; Baker, N.A., PDB2PQR: expanding and upgrading automated preparation of biomolecular structures for molecular simulations. Nucleic. Acids. Res. 2007, 35, W522-525.

35. Li, P.; Merz, K.M. MCPB.py: A Python Based Metal Center Parameter Builder. J. Chem. Inf. Model. 2016, 56, 599-604.

36. Mark. P.; Nilsson, L., Structure and Dynamics of the TIP3P, SPC, and SPC/E Water Models at 298 K. J. Phys. Chem. 2001, 105, 9954-9960.

37. Case, D. A.; Cheatham, T. E.; Darden, T.; Gohlke H.; Luo, R.; Merz, K. M.; Onufriev, A.; Simmerling, C.; Wang, B.; and Woods. R. J., The Amber biomolecular simulation programs. J. Comput. Chem. 2005, 26, 1668-1688.

38. Maier, J.A.; Martinez, C.; Kasavajhala, K.; Wickstrom, L.; Hauser, K.E; Simmerling, C., ff14SB: Improving the Accuracy of Protein Side Chain and Backbone Parameters from ff99SB. J. Chem. Theory. Comput. 2015, 11, 3696-713.

39. Darden, T.; York, D.; Pedersen, L., Particle mesh Ewald: An $N \cdot \log (N)$ method for Ewald sums in large systems. J. Chem. Phys. 1993, 98, 10089.

40. Phillips, J. C.; Braun, R.; Wang, W.; Gumbart, J.; Tajkhorshid, E.; Villa, E.; Chipot, C.; Skeel, R. D.; Kalé, L.; and Schulten, K., Scalable molecular dynamics with NAMD. J. Comput. Chem. 2005. 26, 1781-1802

41. Humphrey, W.; Dalke, A.; and Schulten, K., VMD-Visual Molecular Dynamics. $J$. Molec. Graphics, 1996, 14, 33-38.

42. Grant, B.J.; Rodrigues, A.P.C.; El Sawy, K.M., McCammon, J.A.; Caves, L.S.D., Bio3D: An $\mathrm{R}$ package for the comparative analysis of protein structures. Bioinformatics. 2006, 22, 2695-2696.

43. One of the Reviewers of the current work has brought this insight. 